\title{
相变蓄能建筑围护结构热性能研究进展
}

\author{
王馨, 张寅平 ${ }^{*}$, 肖伟, 曾若浪, 张群力, 狄洪发
}

清华大学建筑技术科学系, 北京 100084

* 联系人, E-mail: zhangyp@mail.tsinghua.edu.cn

2008-09-01 收稿, 2008-11-17 接受

国家“十一五”科技支撑计划资助项目(编号: 2006BAA04B02, 2006BAJ02A09)

摘要 改进建筑围护结构热性能是建筑节能的重要途径. 相变蓄能建筑围护结构对于有效利用可 再生能源, 降低建筑运行能耗, 节省运行费用, 提高建筑热舒适度, 减小环境污染和温室气体排 放具有重要意义. 介绍了理想节能建筑围护结构概念, 以此指导建筑围护结构材料遴选和热性能 设计; 对国内外相变建材和相变蓄能围护结构的研究进展进行了综述, 并指出了需要解决的问题.

关键词 相变材料 蓄能 热质 围护结构 建筑节能
随着我国经济发展和人民生活水平的提高, 我 国建筑能耗增长迅速, 统计数据显示, 2004 年我国建 筑总面积为 389 亿平方米. 建筑能耗占社会总能耗的 $25.5 \%{ }^{[1]}$, 建筑节能刻不容缓. 在国务院批准的《节能 中长期专项规划》中, 建筑节能已被列为节能的重点 领域.

建筑围护结构作为室内外环境的分界面, 在提 供安全、隐私、出入口和视域的同时, 调整着各种形 式的能量(光、热、声、湿等)流通. 具体说来, 围护 结构在建筑使用过程中承担的功能主要有视野、采 光、遮阳与隔热、保温(散热)、通风和隔声等六大方 面. 改进建筑围护结构形式与热性能, 是建筑节能的 重要途径.

人类社会在几千年的发展过程中，绝大部分时 间都采用被动式手段来调节室内热环境, 如冬季利 用太阳能采暖, 夏季利用自然通风“空调”。被动式调 节的特点是依靠建筑围护结构自身的调温特性, 充 分利用外环境自然调节改善室内环境. 这类传统建 筑大都使用重质材料建造, 热惯性较大, 能有效抑制 室内温度波动, 冬暖夏凉, 如陕北地区的窑居建筑 ${ }^{[2]}$. 目前现代建筑尤其是高层建筑中, 轻质围护结构应 用越来越广泛, 可大大减轻建筑结构自重, 且大都具 有良好的保温隔热性能. 同时, 出于景观和自然采光
的需要, 现代建筑经常采用大面积的透明围护结构, 冬季利用从玻璃进入室内的太阳辐射热采暖. 但是 由于轻质围护结构热容小, 易造成室内温度白天过 高、夜间过低, 昼夜波动大, 热舒适性差. 如何充分 合理地利用建筑围护结构热质(thermal mass), 依靠 建筑围护结构自身的调温特性, 充分利用可再生自 然能源而不采用(或少采用)附加能源达到人体热舒 适要求, 对有效利用清洁廉价的可再生能源, 降低建 筑运行能耗, 节省运行开支, 减小环境污染等具有重 要意义. 为此, 国际能源署(IEA)下属机构ECES (Energy conservation through energy storage)已将储热在 建筑中的应用作为主要的研究方向.

\section{1 理想节能建筑围护结构}

影响室内空气温度的主要因素有: ( i ) 室外环 境参数(室外温度、风速、辐照、天空温度); ( ii ) 围 护结构热性能(热阻特性、热容特性); (iii) 室内热扰 与换气; (iv) 室内辅助调温手段(如空调或采暖)等. 在其他条件给定的情况下(气象条件、换气次数、房 间尺寸和墙体厚度等), 室温和建筑围护结构材料特 性紧密相关.

\section{1 理想节能建筑概念}

图 1 为某建筑全年室内外空气温度变化示意图, 
由于围护结构的蓄热, 与环境温度相比, 室温的日波 动和年波动都有衰减和延迟现象. 文献[3]提出了理 想节能建筑围护结构概念, 即如果建筑围护结构的 蓄热和隔热性能对室外温度波动产生合适的衰减和 延迟作用, 不用采暖和空调即可使室温处于舒适温 度区, 这样的建筑称为被动式理想节能建筑, 这样的 围护结构称为被动式理想节能建筑围护结构.

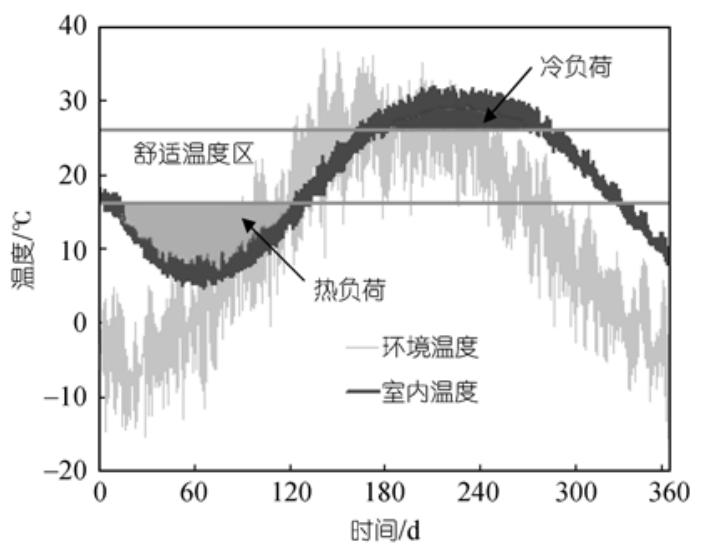

图 1 某建筑室内外温度示意图

一般说来, 建筑热舒适性和能耗密切相关, 图 2 为采用不同围护结构的建筑能耗与舒适性的关系示 意图. 可见, 理想节能建筑意味着仅用自然能源即可 使建筑位于舒适区, 即建筑围护结构性能曲线必须 能够落在区域 A 中. 要达到这个目标, 除了通过各种 方式充分利用太阳能、夜间冷风能等自然能源外, 还 需使用热性能好的建筑围护结构.

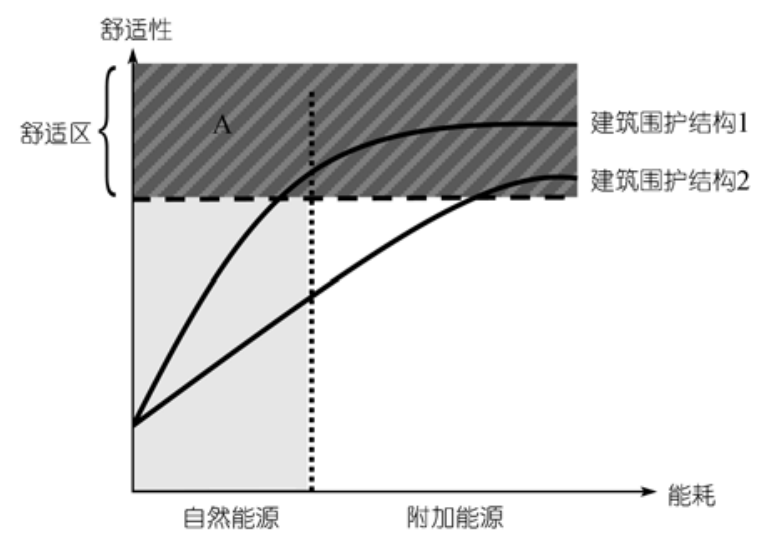

图 2 建筑能耗与舒适性关系示意图

传统建筑围护结构热性能分析一般采用正问题 分析方法, 即在已知建筑围护结构形式、材料和热物
性的前提下, 分析其热性能, 并以此为基础, 得到相 应的采暖空调负荷, 确定采暖空调方式. 这种正问题 的分析方法难以确定建筑的最佳围护结构形式、材料 的最佳热物性和采暖空调的最佳方式. 而理想节能 建筑从反问题角度研究如何依靠建筑围护结构自身 热质的调温特性, 充分利用自然调节即可再生自然 能源以达到不采用(或少采用)附加能源满足人体热 舒适的要求, 通过建筑围护结构热质线性和非线性 节能调温原理分析, 确定建筑的最佳围护结构形式、 建材的最佳热物性及适用条件和范围, 是建筑围护 结构和建材的热物性设计与研制的一个新的尝试方 向.

\section{2 理想节能建筑评价参数}

为了评价无采暖空调建筑的热舒适性, 文献 [3] 以房间全年的自然室温为对象, 定义了夏季过热不 舒适度 $I_{\mathrm{sum}}$ 和冬季过冷不舒适度 $I_{\text {win }}$.

$$
\begin{aligned}
& I_{\text {sum }}=\int_{\text {year }}\left(T_{\text {in }}-T_{\mathrm{H}}\right) \mathrm{d} \tau \quad T_{\text {in }}>T_{\mathrm{H}}, \\
& I_{\text {win }}=\int_{\text {year }}\left(T_{\mathrm{L}}-T_{\text {in }}\right) \mathrm{d} \tau \quad T_{\text {in }}<T_{\mathrm{H}},
\end{aligned}
$$

其中, $T_{\text {in }}$ 为室内操作温度, $T_{\mathrm{H}}$ 和 $T_{\mathrm{L}}$ 分别为热舒适区温 度的上下限.

可见, $I_{\text {sum }}$ 和 $I_{\text {win }}$ 越小房间越舒适. 当 $I_{\text {sum }}$ 和 $I_{\text {win }}$ 等于或接近 0 时, 不需采暖空调即可维持热舒适性, 即为被动式理想节能建筑.

\section{3 建筑围护结构热性能}

非透明围护结构控制方程可表示为

$$
\rho \frac{\partial H}{\partial \tau}=k \frac{\partial^{2} T}{\partial x^{2}},
$$

其中, 线性常物性材料的焓表示为 $H=\int_{T_{0}}^{T} C_{p} \mathrm{~d} T$, 非线 性相变材料的焓表示为 $H=\int_{T_{0}}^{T_{\mathrm{L}}} c_{p, \mathrm{~s}} \mathrm{~d} T+\int_{T_{\mathrm{L}}}^{T_{\mathrm{H}}} c_{p, \mathrm{~m}} \mathrm{~d} T+$ $\int_{T_{\mathrm{H}}}^{T} c_{p, 1} \mathrm{~d} T, T$ 为材料在 $\tau$ 时刻的温度, $T_{0}$ 为焓值为 0 时 的温度, $T_{\mathrm{L}}$ 到 $T_{\mathrm{H}}$ 为相变温度范围, $\rho$ 为密度, $k$ 为导热 系数, $c_{p}$ 为比热.

围护结构控制方程结合边界条件和初始条件以 及室内空气热平衡方程, 构成非透明围护结构焓法 传热模型. 由此可得到 ${ }^{[3]}$

$$
\rho c_{p}=f\left(A C H, t_{\mathrm{in}}, k\right),
$$

其中, $A C H$ 是室内换气次数. 
Zhang等人 ${ }^{[3]}$ 利用此模型对北京地区给定常物性 材料对象房间进行了研究, 在外墙 $k=0.05 \mathrm{~W} /\left(\mathrm{m} \cdot{ }^{\circ} \mathrm{C}\right)$, 内墙 $k=0.5 \mathrm{~W} /\left(\mathrm{m} \cdot{ }^{\circ} \mathrm{C}\right)$ 情况下, 当 $\rho c_{p}$ 大于 $50 \mathrm{MJ} /\left(\mathrm{m}^{3}\right.$. $\left.{ }^{\circ} \mathrm{C}\right)$ 时, $I_{\text {win }}$ 接近 0 , 即冬季可达到被动式理想节能建筑; 当 $\rho c_{p}$ 大于 $100 \mathrm{MJ} /\left(\mathrm{m}^{3} \cdot{ }^{\circ} \mathrm{C}\right)$ 时, $I_{\text {sum }}$ 和 $I_{\text {win }}$ 都接近 0 , 即 全年可达到被动式理想节能建筑.

现有建材多为常物性材料, 其热容 $\rho c_{p}$ 的值在 $0 \sim 4.0 \mathrm{MJ} /\left(\mathrm{m}^{3} \cdot{ }^{\circ} \mathrm{C}\right)$ 的范围内 ${ }^{[3]}$, 远达不到被动式理想 节能建筑围护结构所要求的热容. 而相变材料在相 变过程中可吸收或放出大量的热量, 在相变温度附 近的等效比热很大, 其热性能更接近理想节能建筑 要求.

\section{2 相变材料与相变建材}

常见的相变蓄能材料种类及特点见表 $1^{[4]}$.

目前适用于建材用的商业化相变材料产品大都 为石蜡和水合盐. Khudhair等人吕给出了建筑蓄能常 用的无机和有机相变材料. 一般说来, 无机相变材料 价格较便宜, 有机相变材料价格较贵. 由于传统固液 相变材料使用时需封装, 在建筑中应用较困难, 不少 研究者对材料进行了研究与改进. Hawlader等人 ${ }^{[6]}$ 研 究了各参数对微封装相变材料性能(如封装效率、蓄 放热量等)的影响. Hawes和Feldman等人 $[7,8]$ 综述了有 机相变材料在各种建筑水泥中的吸收特性和吸收机 理, 分析了温度、湿度、黍性、吸收面积和压力等因 素对吸收特性的影响. Hawes等人 ${ }^{[9,10]}$ 研究了有机相 变材料在各种建筑水泥中的稳定性, 指出相变材料 渗入水泥中, 能显著提高墙体的储热能力, 但其长期 稳定性和现有水泥的吸收特性有待进一步改善. 美国 学者 ${ }^{[11]}$ 根据Florida的气候条件研究了几种可用于石 亳板的储热材料, PCM主要是硬脂酸甲脂、硬脂酸丁 脂、棕㭣酸甲脂以及它们的混合物, 这些物质有较高
的相变潜热, 可以获得一系列不同相变温度的PCM. 但是在比较炎热的气候条件下, 它们的相变温度范 围仍然不能很好地适应人居建筑所需要的温度. 德 国BASF公司(http://www.basf.de/dispersion)将石蜡封 装在微胶囊中, 研制出石蜡砂浆, 并已将这种砂浆用 于房屋的内墙表面上, 作为室内的冬季保温和夏季 制冷的材料, 这样的设计可以减少室内温度波动, 使 室内保持良好的热舒适度, 减少空调系统的设备容 量, 转移用电负荷. 砂浆内含 $10 \%$ 25\%(质量分数)的 石蜡微胶囊, 也就是说, 每平方米的墙面就含有 750 $1500 \mathrm{~g}$ 的石蜡微胶囊. 每 $2 \mathrm{~cm}$ 厚的此种砂浆的 蓄热能力相当于 $20 \mathrm{~cm}$ 厚的砖木结构.

近几年出现的一种定形相变材料(shape-stabilized $\mathrm{PCM})^{[12 \sim 14]}$, 是由相变材料(芯材)和高密度聚乙烯作 为支撑材料(囊材)构成. 在微观尺度内高密度聚乙烯 将定形相变材料包裹交联起来, 由于囊材的熔点较 高形成空间网状结构, 对作为芯材的相变材料起着 微封装和支撑作用. 当工作温度低于高分子囊材的 熔点时, 即使相变材料芯材发生固-液相转变过程, 相变材料依旧被包裹在囊材中, 可以维持形状不变. 此材料可制成粒状、棒状, 也可制成板材, 适合在建 筑中直接应用或者与混凝土掺混使用.

由上可见, 目前已经形成了一些比较成熟的相 变材料与普通建筑材料的复合使用方式. 但仍存在 一些问题, 如: 相变材料成本过高、相变材料与普通 建材复合使用过程中存在力学强度降低问题、阻燃问 题、相变复合建材的规模化生产工艺问题等.

\section{3 蓄能式相变建筑围护结构}

将相变材料复合到现有建材中, 可以在建筑承 重增加较小的条件下有效增大建筑热惯性, 减小室

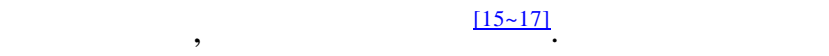

表 1 相变材料分类及其优缺点比较 $[4]$

\begin{tabular}{|c|c|c|}
\hline 分类 & 无机材料 & 有机材料 \\
\hline 种类 & 结晶水合盐类、熔融盐类、金属或合金类等结晶水合盐类 & $\begin{array}{l}\text { 高级脂肪烃类、脂肪酸/酯类或盐类、醇类、芳香烃类、芳香 } \\
\text { 酮类、酰胺类、氟利昂类、多羟基碳酸类等、一些高分子类 }\end{array}$ \\
\hline 优点 & 导热系数大、单位体积的储热密度大、价格便宜、不可燃 & $\begin{array}{l}\text { 在固体状态时成型性较好、一般不容易出现过冷现象和相分 } \\
\text { 离、材料的腐蚀性较小、性能比较稳定、毒性小 }\end{array}$ \\
\hline 缺点 & 过冷、易相分离、腐蚀性大 & $\begin{array}{l}\text { 导热系数小、密度小、熔点较低, 不适于高温场合中应用, 且 } \\
\text { 易挥发、易燃烧甚至爆炸或被空气中的氧气缓慢氧化而老化 }\end{array}$ \\
\hline 性能改进 & $\begin{array}{l}\text { 解决过冷: 加成核剂、冷指法; 解决结晶: 加增稠剂、 } \\
\text { 加晶体结构改变剂、盛装相变材料的容器采用薄层结 } \\
\text { 构、摇晃或摚动 }\end{array}$ & $\begin{array}{l}\text { 通过添加高导热系数材料提高相变材料的导热系数; 通过添 } \\
\text { 加阻燃剂或封装的方法改善相变材料的易燃性能 }\end{array}$ \\
\hline
\end{tabular}


表 2 相变蓄能构件多种应用形式
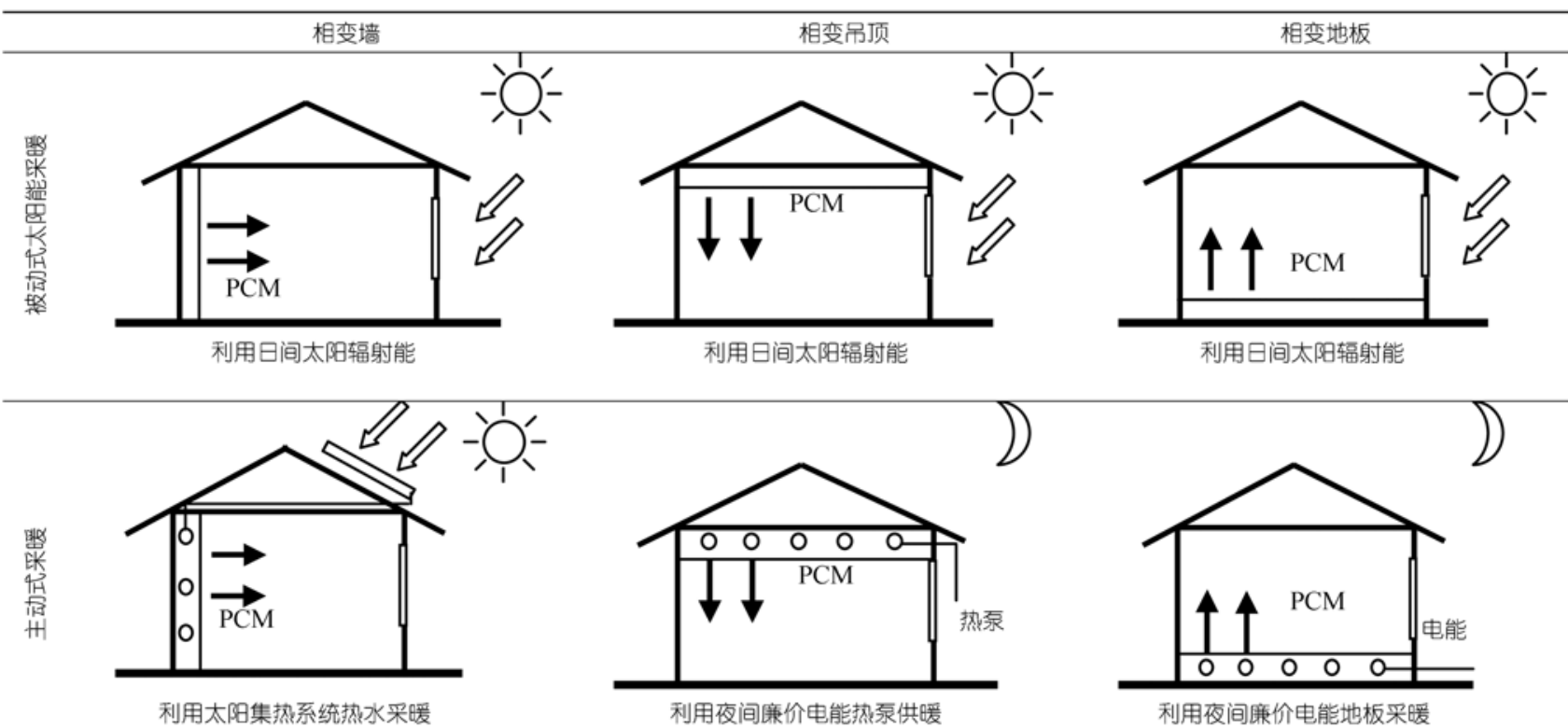

D
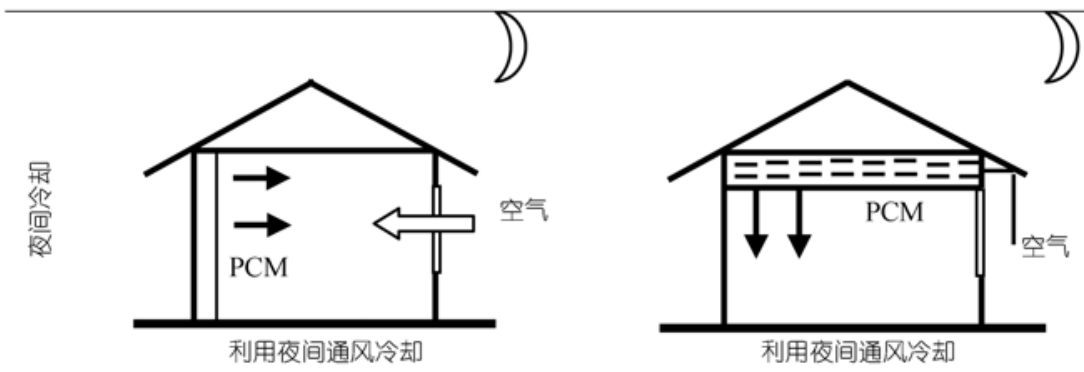

利用夜间廉价电能地板采暖

利用夜间通风冷却

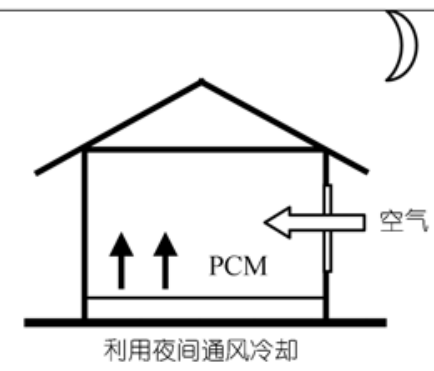

可分为被动蓄能式相变建筑围护结构和主动蓄能式相 变建筑围护结构. 被动蓄能式建筑围护结构主要依靠 室温的变化或接受太阳辐射热流等方式吸收和释放热 量, 主动蓄能式建筑围护结构则把相变材料建筑构件 与采暖空调末端形式有机集成在建筑围护结构内, 相 变材料的吸放热过程可以通过换热装置主动地进行调 节与控制. 表 2 给出了相变蓄能构件的多种应用形式.

合理优化配置建筑围护结构热质和蓄放热能力, 可以解决建筑物能量供需双方在时间和使用强度上 不匹配的问题, 起到如下作用: (i ) 与太阳能或夜间 冷风等蓄热或蓄冷方式结合, 充分利用可再生能源, 降低建筑能耗; (ii ) 提高室内热环境稳定性, 减少室 内温度波动, 增加室内环境舒适性; (iii) 对建筑的采 暖空调负荷起到削峰和延迟作用, 从而有助于减少 空调机组和供暖设备的装机容量.

\section{1 被动蓄能式相变建筑围护结构}

诸多学者对无内热源的被动蓄能式相变建筑围 护结构(墙体、地板、吊顶)的蓄能调温特性和应用可 行性进行了研究.

Chandra等人 ${ }^{[18]}$ 计算了相变储能墙板的厚度对室 内温度变化的影响, 以及各类构件如屋顶、南墙、北 墙和西墙各处热流量的变化. 计算结果显示 $5 \mathrm{~cm}$ 的 相变储能墙板的热效果与 $23 \mathrm{~cm}$ 的混凝土墙板相当; 并且相变储能墙板用在南面墙体效果最好.

Neeper等人 ${ }^{[19]}$ 对相变墙体的一维非稳态传热过程 进行了理想简化, 忽略相变墙体的热阻影响, 分析室 内温度在周期性波动和实际室温波动工况条件下， 相变墙体的蓄传热能力. 结果表明即使墙体没有导 热热阻, 由于墙体表面对流热阻和外界条件的限制, 墙体的最大吸热量在 $300 ~ 400 \mathrm{~kJ} / \mathrm{m}^{2}$; 最佳相变温度 
为房间平均舒适温度. 他建立的这种方法为优化选 取相变温度和估算相变材料在不同使用量下的使用 效果提供了参考.

林坤平 ${ }^{[20]}$ 建立了相变墙体蓄热过程和放热过程 的简化分析模型, 得出了相变外墙的蓄传热特性. 研 究发现和普通内墙传热过程相比, 相变外墙的蓄传 热过程更为复杂, 传统的墙体的热性能评价参数(衰 减系数和延迟时间)不能够完全反映相变墙体的传热 特性, 为此补充了热流修正因子和相变传热过程时 间两个参数用于反映相变墙体的传热过程特性.

Peippo等人 ${ }^{[21]}$ 在美国威斯康星州麦迪逊地区建 立一座 $120 \mathrm{~m}^{2}$ 的轻质被动式太阳房(具有较好保温性 能和较大的南向窗户)中, 将相变材料墙板作为该房 间的热存储构件. 研究发现该房间在一年内可以节省 3 GJ的热量或节省15\%的年运行能耗费用, 同时发现 日存储过程中的最优相变温度比室内温度高 $1 \sim 3^{\circ} \mathrm{C}$.

美国Oak Ridge实验室的Stovall和Tomlinson ${ }^{[22]}$ 的 分析表明相变石亳墙板的成本比普通石膏墙板增加 约 1.30 美元 $/ \mathrm{m}^{2}$. 对于北美常见的房子如果采用相变 石膏墙板用于辅助冬天采暖, 在经济性上以热泉作 为比较, 采用相变墙的投资回收期约为 5 年. 通过模 拟得出, 对于类似美国田纳西州气候类型的地区, 使 用相变墙板能使采暖设备容量选型减少 $1 / 3$, 而对于 类似丹佛气候类型的地区, 使用相变墙板能使采暖 设备容量选型减少 $1 / 2$.

Ismail与Castro ${ }^{[23]}$ 在 1997 年采用商用多元醇的混 合物制作 $\mathrm{PCM}\left(\right.$ 相变温度是 $\left.23 \sim 26^{\circ} \mathrm{C}\right)$, 建造了一个 $1.25 \mathrm{~m}($ 长 $) \times 1.8 \mathrm{~m}($ 宽 $) \times 1.7 \mathrm{~m}$ (高)的坡屋顶实验测试房, 经过两年测试, 并利用一维非稳态传热模型进行了 数值模拟. 结果表明采用PCM后, 空调负荷可以减少 $25 \%$ 30\%, 并可以明显转移峰值空调负荷.

加拿大 Concordia 大学的建筑研究中心的 Athienitis等人 ${ }^{[24]}$ 在被动式太阳房中使用相变墙, 结果 显示房间的温度在白天比常规墙板房间温度低 $4^{\circ} \mathrm{C}$, 而夜间其放热可以延续 $7 \mathrm{~h}$ 以上, 夜间相变墙板的表 面温度可比普通墙板高 $3.2^{\circ} \mathrm{C}$, 相变材料凝固过程放 出的热量相当于总供热负荷的 $15 \%$, 可在很大程度 上改善房间热舒适性.

Kang等人 ${ }^{[25]}$ 提出了一种结合夜间通风的新型接 能系统, 堆积床式相变吊顶系统(NVPsystem), 其梡 换热面积大, 蓄/放热效率高, 夏季降温效果好.

林坤平等人 ${ }^{[26,27]}$ 分析了采用相变墙建筑适用的
气候条件, 并通过数值方法模拟和评价了相变墙房 间在我国不同气候地区的使用效果, 说明了相变墙 建筑在我国不同地区使用的优点和局限性, 并对给 定的气象条件和相变墙房间, 讨论了夏季“空调” 型 相变墙的优化设计方法.

Zhang等人 ${ }^{[28]}$ 设计了一种相变节能型墙体, 将石 蜡材料经过宏观封装成圆柱嵌入在框架墙体中, 现 场测试结果表明该墙体用于民用建筑中可以降低墙 体壁面峰值热流的 $38 \%$, 将日间的部分负荷转移到 夜间, 维持相对较稳定的室内温度, 减少设备的启停 次数.

张寅平等模拟研究了直接受益式定形相变墙板 和地板房间的热性能 $[29,30]$, 从相变温度、潜热、导热 系数、对流换热系数、相变板厚度、相变板放置位置、 保温形式等几个方面分析了其使用效果, 研究结果 表明对流换热系数对使用效果影响很大. 相变温区 越窄, 使用效果越好; 相变石膏板和定形相变墙板分 别使室温波动减小了 $46 \%$ 和 $56 \%{ }^{[31]}$.

综上所述, 相变蓄能建筑围护结构能增加轻质 建筑围护结构的热容, 增加外围护结构的热惰性, 减 少室内温度波动, 降低室内空调负荷.

\section{2 主动蓄能式相变建筑围护结构}

主动蓄能式建筑围护结构内部可布置一些热源 或换热装置, 如预设电热型电缆或电热膜、预埋水管 或风管等, 有利于充分利用可再生能源, 节省运行费 用, 增大室内热舒适性. 从蓄存能量的用途角度, 可 分为主动式相变蓄热和主动式相变蓄冷.

(i) 主动式相变蓄热. 主动式相变蓄热研究主 要集中在地板采暖上, 根据采暖热源不同, 可分为相 变蓄能电采暖和相变蓄能太阳能采暖; 根据传热介 质不同, 又可分为直接相变蓄能电采暖、相变蓄能热 水采暖和相变蓄能热风采暖.

(1) 直接相变蓄能电采暖. 直接相变蓄能电采 暖由于简单易行, 受到较多学者的关注.

Barrio等人 ${ }^{[32]}$ 采用NPG固固相变材料作为地板 蓄能材料, 利用夜间低谷廉价电采暖, 显示了固固相 变材料的可行性. Athienitis等人 ${ }^{[33,34]}$ 研究了混凝土蓄 热地板电采暖系统的热性能和使用效果, 同混凝土 相比, 相变材料具有单位蓄能密度大的特点.

叶宏等人 ${ }^{[35 ~ 38]}$ 提出将定形相变材料与电缆加热 的地板电采暖结合使用, 并对该系统进行了实验和 
模拟研究. 研究结果表明熔点在 $32^{\circ} \mathrm{C}$ 左右的定形相 变材料是该系统较理想的贮热材料, 全天室内空气 温度可维持在 $21 \sim 25^{\circ} \mathrm{C}$, 具有很好的热舒适性; 该系 统在实行昼夜电价分时制的地区具有较好的应用前 景. 罗晓喜等人 ${ }^{[39]}$ 介绍了电加热相变蓄热地板供暖 的优点, 综合考虑室外气象因素、室内辐射特性和相 变材料自身的热物性, 建立了电加热相变蓄热地板 供暖系统的计算模型, 比较了相变蓄热地板与传统 散热器供暖的热舒适性和能耗, 结果表明相变蓄热 地板能够改善室内热舒适性和降低能耗.

林坤平等人 ${ }^{[40 \sim 43]}$ 建立电热膜加热的相变蓄能地 板的一维非稳态传热过程的数学模型, 提出了与夜 间电采暖相结合的新型定形相变材料地板蓄热系统, 开展了定形相变材料蓄能式电热膜地板采暖系统的 实验与模拟研究. 该系统可有效提高和均匀房间温 度, 能把白天负荷全部转移到了夜间, 有效利用夜间 廉价能源, 实现整个采暖季节夜间蓄能日间供暖的 效果. 她们还模拟分析了不同的气候条件下, 相变材 料适当的相变温度和相变材料与地板装饰层之间空气 层的厚度, 并介绍了该系统简单的设计和控制方法.

Zhou等人 ${ }^{[44]}$ 模拟研究了一种结合定形相变蓄能 墙板的混合式供暖系统的使用效果. 辅助热源系统 白天维持室温在 $18^{\circ} \mathrm{C}$ 以上, 夜间满负荷打开使墙板 蓄热. 结果表明, 定形相变板的使用使平段和峰段的 耗电量减小了 $47 \%$, 总能耗减小了 $12 \%$.

由于相变蓄能电采暖消耗的是高品位的电能, 因此只适合于分时电价比较大的地区. 受建筑节能 影响, 鼓励采用低品位的能源进行建筑采暖与空调, 因此利用自然能源的主动式相变建筑围护结构受到 更多的关注.

(2) 相变蓄能热水采暖. 张群力等人 ${ }^{[45,46]}$ 提出 了相变蓄能式低温热水地板采暖系统和吊顶采暖空 调系统, 建立了系统在室内热环境稳态工况下的传 热过程模型, 通过数值模拟方法分析相变温度对地 板和吊顶表面平均热流和蓄能比的影响, 比较了定 形相变材料与混凝土地板及吊顶热性能差异. 结果 表明, 相变材料蓄能效率比混凝土高 $30 \%$ 40\%, 导 热系数影响蓄热效率与蓄热量, 相变温度和潜热影 响表面热流量的衰减时间.

（3）相变蓄能热风采暖. 在相变蓄能热风采暖方 面, 林坤平等 ${ }^{[47}$ 501 模拟并实验了地板下送风式定形相 变材料蓄能系统. 结果表明, 高峰用电采暖需求可全
部被低谷用电采暖取代, 在采取峰谷电价的地区可以 节省费用. 对于不同的气候条件, 可通过调整相变材 料的相变温度和送风速率使系统达到供暖需求.

(ii) 主动式相变蓄冷. 主动式相变蓄冷研究主 要为结合夏季夜间冷风的相变蓄能式建筑围护结构, 夜间蓄冷供日间空调使用.

康艳兵 ${ }^{[51]}$ 把相变材料制作成堆积床形状吊顶与 夜间通风相结合, 建立数学模型并对此系统在不同地 域的应用效果进行了分析. 结果表明, 在兰州、成都、 太原, 此系统可满足房间舒适性要求; 在乌鲁木齐、 北京、西安, 要根据用户要求确定系统是否可行; 在 广州该系统不可行. 钟志鹏 ${ }^{[52]}$ 研究了夏季结合夜间 通风的相变墙在不同地区的应用效果, 讨论了通风 次数和相变温度的优化设计方法. 结果表明在日差 较大, 平均温度较低的气候条件下, 结合夜间通风的 相变墙可基本取代空调, 防止夏季房间过热.

瑞典 Climator 公司 (http://www.climator.net) 在 Stevenage Borough 政府办公室安装了一套相变材料 蓄能吊顶用于夜间通风冷却, 白天供冷. 测试结果表 明可以降低室内温度 $3 \sim 4^{\circ} \mathrm{C}$. 德国 Dörken 公司 (http://www.doerken.de)于 2003 年将相变材料放置在 房间吊顶上进行夜间通风蓄冷、在白天供冷. 该系统 采用的无机水合盐类型相变材料相变温度为 $22 \sim 24^{\circ} \mathrm{C}$, 潜热为 $158 \mathrm{~kJ} / \mathrm{kg}$, 相变材料用 $30 \mathrm{~cm} \times 15 \mathrm{~cm}$ 的铝袋封 装. 实验结果表明平均室内温度可以降低 $4^{\circ} \mathrm{C}$, 最大 可降低 $5.5^{\circ} \mathrm{C}$.

德国Koschenz和Lehmann ${ }^{[53]}$ 提出了一种应用于 轻质建筑中的主动式相变蓄能石亳吊顶空调系统. 该系统通过冷水毛细管将冷量夜间蓄存入相变石膏 板中, 日间时再释放出冷量, 可以将白天的空调负荷 转移到夜间处理. 该系统可以利用低品位能源进行 空调采暖, 具有较好的应用前景.

\section{4 结论与展望}

通过优化配置建筑围护结构热物性参数, 增强 其衰减和延迟作用, 不使用或少采用采暖和空调就 可达到舒适温度, 从而成为理想节能建筑围护结构. 这就需要从反问题角度出发, 通过简化与建模, 在气 候条件、建筑围护结构和运行工况给定条件下, 得出 不用或少用附加能源时围护结构所需要的临界热性 能, 从而为建材热物性从需求角度提出了改善目标. 现有的常物性建材由于蓄热能力小, 远达不到理想 
节能建筑围护结构要求. 而相变材料在相变过程中 可吸收或放出大量的热量, 在相变温度附近的等效 比热很大, 其热性能更接近理想节能建筑要求.

合理优化配置建筑围护结构热质和蓄放热能力, 可以解决建筑物能量供需双方在时间和使用强度上 不匹配的问题，起到如下作用: (1)与太阳能或夜间冷 风等蓄热或蓄冷方式结合，充分利用可再生能源，降 低建筑能耗; (2)提高室内热环境稳定性, 减少室内温 度波动, 增加室内环境舒适性; (3)对建筑的采暖空调 负荷起到削峰和延迟作用, 从而有助于减少空调机 组和供暖设备的装机容量.
目前需要解决的一些问题有:

(1) 采用反问题思路分析, 藉变分分析方法, 利 用可再生能源和自然条件, 确定建筑的最佳围护结 构形式、建材的最佳热物性及适用条件和范围.

(2) 进一步加强可和常规建材较好结合的相变 材料的研究, 包括力学性能、热性能与阻燃性能等.

(3) 根据实际需求, 建立并完善相变蓄能建筑围 护结构设计原则与方法.

（4）有效准确评价相变建筑构件在房间中的应 用效果和优势, 并提出推广应用的适宜性条件, 包括 经济性指标.

\section{参考文献}

清华大学建筑节能研究中心。中国建筑节能年度发展研究报告 2008. 北京：中国建筑工业出版社, 2008. 4

刘加平，何泉，杨柳，等. 黄土高原新型窑居建筑. 建筑与文化, 2007, 6: 39-41

Zhang Y P, Lin K P, Zhang Q L, et al. Ideal thermal physical properties for free-cooling (or heating) buildings with constant thermal physical property material. Energ Buildings, 2006, 38(10): 1164-1170[DOI]

张群力. 相变蓄能建筑构件蓄能调温特性及其应用基础研究. 博士学位论文. 北京: 清华大学建筑技术科学系, 2007

Khudhair A M, Farid M M. A review on energy conservation in building applications with thermal storage by latent heat using phase change materials. Energ Convers Manage, 2004, 45: 263-275[DOI]

Hawlader M, Uddin M S, Khin M M. Microencapsulated PCM thermal-energy storage system. Appl Energ, 2003, 74(1-2): 195-202[DOI]

Hawes D W, Feldman D, Banu D. Latent heat storage in building materials. Energ Buildings, 1993, 20(1): 77-86[DOD]

Feldman D, Banu D, Hawes D W. Development and application of organic phase change mixtures in thermal storage gypsum wallboard. Sol Energ Mat Sol C, 1995, 36(2): 147-157 [DO]

Hawes D W, Banu D, Feldman D. Stability of phase change materials in concrete. Sol Energ Mat Sol C, 1992, 27(2): 103-118[DO]

Lee T, Hawes D W, Banu D, et al. Control aspects of latent heat storage and recovery in concrete. Sol Energ Mat Sol C, 2000, 62(3): $217-237 \underline{\text { [DOI] }}$

Rudd A F. Phase-change material wallboard for distributed thermal storage in buildings. ASHRAE Transactions, 1993, 99: 339-346

Inaba H, Tu P. Evaluation of thermophysical characteristics on shape-stabilized paraffin as a solid-liquid phase change material. Heat Mass Transfer, 1997, 32(4): 307-312 [DOI]

Xiao M, Feng B, Gong K C. Preparation and performance of shape stabilized phase change thermal storage materials with high thermal conductivity. Energ Convers Manage, 2002, 43(1): 103-108 $\underline{\text { [DOI] }}$

Zhang Y P, Ding J H, Wang X, et al. Influence of additives on thermal conductivity of shape-stabilized phase change materials. Sol Energ Mat Sol C, 2006, 90 (11): 1692-1702[DO]

Zhang Y P, Zhou G B, Lin K P, et al. Application of latent heat thermal energy storage in buildings, state-of-the-art and outlook. Build Environ, 2007, 42: 2197-2209 [DOI]

Zhang Y P, Yang R, Lin K P, et al. Preparation, thermal performance and application of shape-stabilized PCM in energy efficient buildings. Energ Buildings, 2006, 38(10): 1262-1269 [DOI]

周国兵, 张寅平，林坤平，等。定形相变材料咜能在暖通空调领域的应用研究。暖通空调, 2007, 37(5): 27一-32

Chandra S, Kumar R, Kaushik S, et al. Thermal performance of a non-air-conditioned building with PCM thermal storage. Energ Convers Manage, 1985, 25: 15-20[DOI]

Neeper D A. Thermal dynamics of wallboard with latent heat storage. Sol Energy, 2000, 68: 393-403 [DOD]

林坤平. 相变蓄能建筑构件应用原理和效果研究. 博士学位论文. 北京: 清华大学建筑技术科学系, 2006

Peippo K, Kauranen P, Lund P D. A multi-component PCM wall optimized for passive solar heating. Energ Buildings, 1991, 17(4): $259-270 \underline{\mathrm{DO}]}$

Stovall T K, Tomlinson J J. What are the potential benefits of including latent storage in common wallboard. ASME T J Sol Energy Engin, 1995, 117(4): 318-325[ [DOI] 
Ismail K A R, Castro J N. PCM thermal insulation in buildings. Int J Energ Res, 1997, 21: 1281-1296[DOI]

Athienitis A K, Liu C, Hawes D, et al. Investigation of the thermal performance of a passive solar test-room with wall latent heat stor-

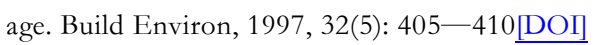

Kang Y B, Jiang Y, Zhang Y P. Modeling and experimental study on an innovative passive cooling system-NVP System. Energ Buildings, 2003, 35(4): 417-425 [DOI]

林坤平，张寅平，江亿．我国不同气候地区夏季相变墙房间热性能模拟和评价. 太阳能学报, 2003, 24(1): 46一 52

林坤平, 张寅平, 江亿. 夏季“空调”型相变墙热设计方法. 太阳能学报, 2003, 24(2): 145-151

Zhang M, Medina M A, Jennifer B K. Development of a thermally enhanced frame wall with phase change materials for on-peak air conditioning demand reduction and energy savings in residential buildings. Int J Energ Res, 2005, 29: 795-809 [DOI]

Zhou G B, Zhang Y P, Lin K P, et al. Thermal analysis of a direct-gain room with shape-stabilized PCM plates. Renew Energ, 2008, 33(6): $1228-1236 \underline{\text { DOI] }}$

Xu X, Zhang Y P, Lin K P, et al. Modeling and simulation on the thermal performance of shape-stabilized phase change material floor used in passive solar buildings. Energ Buildings, 2005, 37: 1084-1091 [DO]

Zhou G B, Zhang Y P, Wang X, et al. An assessment of mixed type PCM-gypsum and shape-stabilized PCM plates in a building for passive solar applications. Sol Energy, 2007, 81(11): 1351-1360[DOI]

Barrio M, Font J, Lopez D O. Floor radiant system with heat storage by a solid-solid phase transition material. Sol Energ Mat Sol C, 1992, 27(2): 127-133[DOI]

Athienitis A K, Chen T Y. Experimental and theoretical investigation of floor heating with thermal storage. ASHRAE Transactions, 1993, 99(1): 1049-1057

Bakos G. Energy management method for auxiliary energy saving in a passive solar heated residence using low cost off peak electricity. Energ Buildings, 2000, 31(3): 237-241피

叶宏, 葛新石, 焦冬生. 带定形 PCM 的相变䛎能式地板辐射采暖系统热性能的数值模拟. 太阳能学报, 2002, 23(4): 482-487 叶宏, 程丹鹏, 葛新石, 等. 定形相变陉能式地板辐射采暖系统数值模型的实验验证及参数分析. 太阳能学报, 2004, 25(2): 189-194

叶宏, 何汉峰, 葛新石, 等. 利用焓法和有效热容法对定形相变材料熔解过程分析的比较研究. 太阳能学报, 2004, 25(4): 488一-491

叶宏, 王军, 庄双勇, 等. 定形相变财能式地板辐射采暖系统的实验研究. 太阳能学报, 2004, 25(5): 651一656

罗晓喜, 张寅平, 林坤平. 电加热相变蓄热地板采暖房间热性能数值模拟及分析. 暖通空调, 2004, 34(11): 5一

林坤平，张寅平，狄洪发，等。电加热相变材料蓄热地板采暖的热性能模拟。太阳能学报, 2003, 24(5): 633-637

林坤平, 张寅平, 狄洪发, 等. 定形相变材料蓄热地板电采暖热性能研究. 清华大学学报(自然科学版), 2004, 44(12): 1618一 1621

林坤平, 张寅平, 徐煦, 等. 定形相变材料蓄热地板电采暖热性能实验研究. 高技术通讯, 2005, 15(4): 51一 54

Lin K P, Zhang Y P, Xu X, et al. Experimental study of under-floor electric heating system with shape-stabilized PCM plates. Energ Buildings, 2005, 3: 215-220 [DO]

Zhou G B, Zhang Y P, Zhang Q L, et al. Performance of a hybrid heating system with thermal storage using shape-stabilized phase change material plates. Appl Energ, 2007, 84(10): 1068-1077[DO]

张群力, 狄洪发, 林坤平, 等. 相变蓄能式低温热水地板采暖系统传热性能模拟研究. 工程热物理学报, 2006, 27(4)：641一 643

张群力, 狄洪发, 张寅平, 等. 双层相变材料蓄能型辐射采暖与空调吊顶热性能. 化工学报, 2006, 57(增刊): 74-79

Lin K P, Zhang Y P, Xu X, et al. Modeling and simulation of under-floor electric heating system with shape-stabilized PCM plates. Build Environ, 2004, 39: 1427-1434 [DOD

林坤平, 张寅平, 狄洪发, 等. 地板下送风式相变蓄热电采暖系统. 太阳能学报, 2005, 26(6): 821一824

林坤平, 张寅平, 狄洪发, 等. 地板下送风相变蓄热电采暖系统热性能模拟. 太阳能学报, 2006, 27(8): 737一742

Lin K P, Zhang Y P, Di H F, et al. Study of an electrical heating system with ductless air supply and shape stabilized PCM for thermal storage. Energ Convers Manage, 2007, 48(7): 2016-2024[DOI]

康艳兵. 夜间通风相变咜能吊顶系统研究. 博士学位论文. 北京: 清华大学建筑技术科学系, 2001

钟志鹏. 结合夜间通风的相变墙房间热性能研究. 硕士学位论文. 北京: 清华大学建筑技术科学系, 2001

Koschenz M, Lehmann B. Development of a thermally activated ceiling panel with PCM for application in lightweight and retrofitted buildings. Energ Buildings. 2004, 36: 567-578 [1DOI 